\title{
Review Article \\ Peripheral Biomarkers in Animal Models of Major Depressive Disorder
}

\author{
Lucia Carboni \\ Department of Pharmacy and Biotechnology, Alma Mater Studiorum University of Bologna, Via Irnerio 48, 40126 Bologna, Italy \\ Correspondence should be addressed to Lucia Carboni; lucia.carboni4@unibo.it
}

Received 28 November 2012; Accepted 31 January 2013

Academic Editor: Daniel Martins-de-Souza

Copyright (C) 2013 Lucia Carboni. This is an open access article distributed under the Creative Commons Attribution License, which permits unrestricted use, distribution, and reproduction in any medium, provided the original work is properly cited.

\begin{abstract}
Investigations of preclinical biomarkers for major depressive disorder (MDD) encompass the quantification of proteins, peptides, mRNAs, or small molecules in blood or urine of animal models. Most studies aim at characterising the animal model by including the assessment of analytes or hormones affected in depressive patients. The ultimate objective is to validate the model to better understand the neurobiological basis of MDD. Stress hormones or inflammation-related analytes associated with MDD are frequently measured. In contrast, other investigators evaluate peripheral analytes in preclinical models to translate the results in clinical settings afterwards. Large-scale, hypothesis-free studies are performed in MDD models to identify candidate biomarkers. Other studies wish to propose new targets for drug discovery. Animal models endowed with predictive validity are investigated, and the assessment of peripheral analytes, such as stress hormones or immune molecules, is comprised to increase the confidence in the target. Finally, since the mechanism of action of antidepressants is incompletely understood, studies investigating molecular alterations associated with antidepressant treatment may include peripheral analyte levels. In conclusion, preclinical biomarker studies aid the identification of new candidate analytes to be tested in clinical trials. They also increase our understanding of MDD pathophysiology and help to identify new pharmacological targets.
\end{abstract}

\section{Major Depressive Disorder}

Major depressive disorder (MDD) is a severe disease that is widely diffused all over the world, exerting a heavy toll in terms of morbidity and mortality $[1,2]$. In Europe, mood disorders are the second most frequent group of mental disorders, dominated by MDD, which affects $7 \%$ of the population [2]. MDD is one of the most important contributors to overall burden of disease expressed as DALY (disability as adjusted life years lost), an indicator including the number of years lost due to ill-health, disability, or early death [2]. In addition to the augmented risk of suicide, mood disorders are associated with increased risk of cardiovascular and metabolic diseases $[3,4]$. Affected patients experience heavy impact on everyday life, due to the characteristic of the symptoms that influence the ability to enjoy life.

The diagnosis of MDD as based on the Diagnostic and Statistical Manual of Mental disorders [5] requires the occurrence of a set of symptoms, which seldom present all together. In addition to the main features of MDD, including depressed mood, loss of interest, and inability to experience pleasure, the diagnosis requires the presence of at least four symptoms among feeling of worthlessness, thoughts of death, inability to concentrate, fatigue, psychomotor retardation or agitation, sudden weight loss or gain, and sleep disturbances in either direction [5].

Although a single episode of depression may occur in a lifetime, a frequent development of the disease is towards a recurring disorder with frequent relapses intermingled with intervals of well-being, therefore displaying a tendency for chronicity.

\section{Therapeutic Options for MDD}

Therapeutic options to treat MDD are currently available. They consist of pharmacotherapies, psychotherapies, and physical therapies. Pharmacotherapies are mainly based on monoamine regulation, due to the fortuitous discovery in the 1950s that an antituberculotic medicine endowed with the ability to inhibit monoamine oxidases could improve 
patients' mood. Subsequently, therapeutic efficacy could be demonstrated for numerous compounds that shared the ability to block monoamine reuptake within the presynaptic terminal $[6,7]$. Firstly developed tricyclic antidepressants could not discriminate among monoamine transporters; moreover, they also bound to other neurotransmitter receptors. Successive agents displayed selectivity towards the serotonin transporter (serotonin-selective reuptake inhibitors), thus improving the safety profile and reducing the number of untoward effects [6]. Indeed, increased monoamine concentrations in the synaptic cleft (due to reduced monoamine degradation with monoamine oxidase inhibitors or to reduced presynaptic reuptake with tricyclic antidepressants or serotoninselective reuptake inhibitors) mediate the mechanism of action of almost all efficacious antidepressants, although a melatonergic agonist was recently authorised [6]. Differences among available medications lie on the relative impact exerted on each monoaminergic neurotransmitter and on the side effect profile. Therefore, it can be assumed that the same brain circuits are likely to be affected by all existing treatments.

Moving towards additional treatment options, cognitive behaviour psychotherapy has demonstrated efficacy in mild as well as severe depression, with a lower relapse rate as compared to pharmacotherapy [8]. Interpersonal psychotherapy proved superior efficacy as compared to placebo treatment, displaying similar efficacy and acceptability as pharmacological treatments [9]. Therapeutic options based on physical treatments chiefly include electroconvulsive therapy, repetitive transcranial magnetic stimulation, exercise, and sleep deprivation. The efficacy of these interventions has been demonstrated in various instances [10-13].

\section{Unmet Medical Needs}

Despite the above-mentioned therapies, the need for new medicines is still substantial. The efficacy of available therapies has been demonstrated with respect to no interventions, especially in case of severe depression [14]; nevertheless, a relatively large number of patients do not respond to treatments [15]. Moreover, a number of symptoms may not be adequately resolved also in patients which experience an overall good therapeutic response. In addition, although the safety profile of recent agents is quite superior in comparison to older medicines, the incidence of side effects, such as sexual dysfunctions, may cause therapy discontinuation, especially in younger patients. Therefore, considerable efforts are dedicated to the research of new therapeutic agents addressing different neurobiological targets, with the hope of overcoming the aforementioned issues $[7,16]$. Unfortunately, approaches aimed at identifying therapies based on entirely different mechanisms have not been successful up to now [17], and pharmaceutical companies are disengaging from this disease area, which is perceived as highly risky.

One reason of the difficulties met in discovering new medicines is the insufficient understanding of the neurobiological basis of the disease [18-20]. It seems likely that MDD is a constellation of diseases merging one into the other, which can be fruitfully split into endophenotypes [21].

Several pieces of evidence support the notion that the occurrence of environmental challenges, often in the form of stressful experiences, needs to be associated with a preexisting genetic predisposition to bring about the disease [22]. Different mechanisms have been proposed to explain the pathophysiological basis of MDD from a neurobiological point of view $[18,22]$. They comprise hypotheses based on monoaminergic deficiency, hypothalamicpituitary-adrenal (HPA) axis dysregulation, neurogeneticneurotrophic-growth factor impairment, metabolic disturbances, circadian rhythm desynchronization, and inappropriate stimulation of the immune system [18-20, 23-26]. This multiplicity is likely to be explained by the fact that several of them are intertwined instead of mutually exclusive (e.g., [2730]). Moreover, it is probable that different endophenotypes correlate with different neurobiological adaptations. From this point of view, a lack of criteria to select appropriate patients may contribute to the difficulties experienced in the quest for new medicines that rely on different mechanisms of action as compared to monoamine regulation.

\section{Identification of Clinical Biomarkers}

In this picture, MDD biomarkers in human patients are needed to aid the diagnosis, to guide among therapeutic options, and to support the discovery and development of new medicines [31, 32]. Clinical biomarkers could provide the psychiatrist with an objective diagnostic tool as compared to the current practice of relying on subjective interviews. Moreover, diagnostic biomarkers could attribute specific patients to defined endophenotypes, improving the chance of adopting suitable antidepressant treatments (e.g., pharmacogenetic markers: [33]). Another field for biomarker use is in the area of drug discovery. Current therapies often reach their results after several weeks, although recent literature reported that reduced time frames could be sufficient $[34,35]$. Biomarkers reliably indicating the likelihood of response to therapy could ease the discovery of potential new treatments.

Investigations of new clinical biomarkers for MDD are mainly based on brain neuroimaging techniques; on sleep pattern analyses; on the evaluation of peripheral levels of mRNAs, proteins, or small-molecular weight analytes [36, 37]. The identification of a single peripheral analyte is conceivable as a pharmacogenetic biomarker of response to therapy. In contrast, a panel of peripheral analytes is a more likely candidate as a useful tool to aid diagnosis or therapy.

\section{Biomarker Investigations in Preclinical Models}

The levels of mRNAs, proteins, or small-molecular weight analytes that can be defined as biomarkers of disease are also assessed in preclinical investigations. In this case, the selected biomarker is measured in the tissues of an animal model of MDD, levels are compared with those evaluated in the respective controls, and an association is discovered between 
biomarker levels and diseased state. The main advantage of this kind of investigations lies in the possibility of an accurate control of the experimental conditions, although potentially reducing the significance for human disease.

Most investigations performed in MDD animal models evaluate the levels of mRNAs, protein, or small molecules in brain, as reasonable for a psychiatric disease. This approach will definitely increase our understanding of the neurobiological basis of MDD, with a potential benefit to be reflected also in the biomarker field. Nevertheless, this review is centred on studies addressing only the measure of small molecules, proteins, peptides, or mRNA levels in peripheral tissues such as plasma, serum, blood cells, and urine or faeces. The reason for this selection is that these approaches were considered as more easily translatable to clinical biomarkers. Studies adopting any analytical method and both large-scale and single analyte technologies were analysed.

While clinical studies are designed to investigate the validity of candidate analytes as potential biomarkers, preclinical investigation may have more indirect approaches. Biomarker studies in animals can be divided into four major categories of experimental designs. Firstly, a number of studies investigate if an animal model is relevant for understanding the pathophysiology of MDD: peripheral analytes are measured to compare results with clinical findings. In other investigations, the main objective is identifying new potential biomarkers to be subsequently validated in humans. Other studies examine new putative therapeutic approaches with behavioural tests; the measure of peripheral analytes is included to increase the confidence in the pharmacological target. Finally, putative biomarker levels may be assessed to investigate the molecular mechanism of action of available antidepressants.

\section{Animal Models of MDD}

Preclinical biomarker studies rely on the availability of a suitable animal model of the disease under investigation. An ideal model should reproduce the pathophysiological features of the human disease, display similar signs and symptoms, and respond to efficacious therapies. Given the incomplete comprehension of the neurobiological basis of MDD, the development of an entirely satisfactory animal model is quite unexpected. In addition, even if we could reproduce in animals the exact neurobiological features of MDD, it is nevertheless dubious which phenotype would be produced by pathophysiological alterations which affect distinctively human characteristics (such as the inappropriate sense of guilt that is often experienced by MDD patients). Numerous recent and informative reviews discussing these topics are available [38-44].

Notwithstanding the aforementioned challenges, investigators tried to reproduce in animal models characteristic symptoms of the human disease, such as anhedonia or helpless behaviours. These behaviours are often assessed with tests that quantify the reduction of interest for a pleasurable event (e.g., sucrose intake) or the reluctance to escape from an unpleasant situation (e.g., forced swim test, tail suspension test, and learned helplessness). Models are often based on relevant factors for the neurobiological basis of the disease. Several models rely on the application of a stress procedure since MDD episodes are often brought about by stressful life events, thus supporting an etiological validity of this procedure. The display of depressive-like behaviours is the hallmark of models based on manipulations (e.g., olfactory bulbectomy, [45]) or genetic selections (e.g., [46, 47]). The link between MDD and immune dysregulation has supported a vast number of investigations based on inflammatory models (discussed in [48]).

\section{Preclinical Biomarkers to Investigate the Relevance of the Animal Model}

By the use of MDD models, peripheral biomarkers were measured with different aims in different experimental designs. A number of examples for each category defined in Section 5 and derived from the recent literature are reported in the following sections.

The most widespread use of peripheral biomarkers in animal models of MDD is in the characterisation of that the animal model in itself. Within this group of investigations, there are two principal reasons for measuring a peripheral analyte. On one hand, the aim of the study is to demonstrate the relevance of the model from a neurobiological point of view by reproducing peripheral dysregulations as documented in human patients. Alternatively, the objective is to show that behaviours recalling MDD symptoms can be induced in experimental animals by inducing alterations that also affect peripheral analyte levels. Examples of the former are investigations that report dysregulations of the HPA axis that resemble those observed in depressive patients. Examples of the latter are quite abundant in studies oriented at investigating the association between inflammation and mood disorders. Whereas support for the correlation is derived from studies performed in human patients, animal models are employed to analyse in deeper detail the link between inflammatory cytokines and mood changes in a more controlled environment. In these cases, peripheral levels of cytokines or other inflammation-related analytes are often measured [24, 28, 30, 48, 49].

A few examples of reports that assessed peripheral biomarker levels with the objective of characterising animal models and/or deriving knowledge on the neurobiological basis of MDD are briefly described.

Abe et al. [50] reported the characterisation of an animal model aimed at examining the aetiology of psychiatric disorders caused by prenatal psychological stress. Along with enhanced emotionality in the open field test and depressivelike behaviour in the forced swim test, relevance was given to the observed enhanced activity in the HPA axis.

Animal models of depressive-like behaviours based on the exposure to social stress are deemed to be especially endowed with ethological relevance due to the similarity to naturally occurring situations. To better characterize this model in a number of different experimental settings, peripheral levels of stress hormones, metabolic, immune, 
and neurotrophic analytes were assessed [51-55] in addition to behavioural, metabolic, and physical parameters, as they were considered an important feature for the thorough investigation of the model.

As another example, Bowens et al. [56] developed a model of atypical depression in the rat that largely relied on hyporesponsiveness of the HPA axis, resembling a distinctive feature observed in this subset of patients. The abnormal HPA axis response thus represented an intrinsic, characterising feature of this model, in which anxiety-like and anhedonia-like behaviours were also evaluated. In the same line of reasoning, Touma et al. [57] endeavoured to reproduce features of MDD endophenotypes by focusing on alterations in corticosterone increase in response to a moderate psychological stressor, highlighting the importance of measuring peripheral levels of stress hormones (although in faeces to avoid disturbing the animals). Therefore, corticosterone circadian rhythms were measured to ensure that they reproduced the same abnormalities observed in patients. Additionally, Blugeot et al. [58] explored the preexisting vulnerability that predisposes an individual to develop a MDD episode after stressful life events, in the complex interplay between genetic and environmental factors. To this aim, they identified a subset of animals that displayed a specific molecular response to a priming stressful event that helped predicting a depressivelike response to a successive chronic mild stress protocol. Among other features, it is noteworthy that the association of low Brain-derived Neurotrophic Factor (BDNF) with normal corticosterone serum concentrations was indicated as a putative predictive biomarker of vulnerability to depression.

The correlation between metabolic disorders and major depression was thoroughly investigated in chronic stress models $[59,60]$, in which peripheral levels of leptin were evaluated. Studies by $\mathrm{Lu}$ et al. [59] supported a role for impaired leptin production in depression-like phenotypes induced by chronic stress, suggesting antidepressant activity of leptin. Subsequent findings [60] indicated that decreases in leptin and melanocortin signalling may represent compensatory responses to manage chronic stress at the expense of metabolic derangements.

D'Audiffret et al. [61] applied the unpredictable chronic mild stress model in mice to interrogate the association between depressive disorders and peripheral vascular disease. To assess major contributing mechanisms underlying the changes in vascular reactivity in this stress model, markers of inflammation (TNF- $\alpha$, IL-1 $\beta$, C-reactive peptide, and IL-10), an index of insulin resistance, and a plasma marker of oxidant stress (nitrotyrosine) were measured in addition to vascular reactivity. The findings suggest that insulin resistance, inflammation, and hypertension associated with the exposure to unpredictable chronic mild stress do not play a major role as predictors of vascular dysfunction. As another example, to investigate the mechanism of the association between increased ratio of plasma kynurenine to tryptophan and depressive symptoms, measures of the levels of these analytes were carried out alongside behavioural tests in animal models based on the acute activation of the peripheral innate immune system $[62,63]$.
To investigate the role played by the neurotrophic factor BDNF in MDD, a chronic stress model was implemented by Naert et al. [64]. To validate the selected model, a physiological and behavioural characterization was performed, including the impact on HPA axis activity and reactivity, such as hypothalamic activation and increase in ACTH and corticosterone plasmatic concentrations, since these modifications have been associated with depression in humans. Cirulli et al. [65] tested the hypothesis that early maternal deprivation would affect peripheral levels of neurobiological mediators of depression in peer-reared female and male rhesus macaques to model sex differences involved in different susceptibility to mood disorders. Therefore, blood levels of BDNF, nerve growth factor, cortisol, and growth hormone were assessed, discovering a sex-selective response in the modulation of these biomarkers.

Moranis et al. [66] investigated the effect of dietary supply of polyunsaturated fatty acid on depressive-like behaviours in adult and aged mice. The levels of IL- 6 and IL-10 cytokines were also assessed both centrally and peripherally since in humans a correlation was reported between the amount of IL6 in blood, quality of life, and neuropsychiatric symptoms in the elderly [67]. Therefore, the assessment of cytokine levels was included to provide insight about a potential mechanism involved in the behavioural changes induced by fatty acid insufficiency, alongside with data generated in humans.

Finally, if a specific dysregulation detected in patients is also reproduced in animal models of disease, this supports the importance of the mechanism in the neurobiological basis of the disorder, as illustrated in investigations about the role played by inflammation in MDD. Likewise, Kronfeld-Schor and Einat [25], in exploring the relationship between circadian rhythms and MDD, suggested that the reported alterations in behavioural, biochemical, and molecular rhythms observed in MDD animal models regardless of the way they were induced supported a relevant role of this system.

\section{Preclinical Studies to Identify Candidate Clinical Biomarkers}

A different scope for measuring peripheral analytes in animal models of MDD is the identification or further validation of new biomarkers to be used in humans. The objective of this kind of investigations is to exploit the advantages of animal models versus human patients. In particular, the relationship between peripheral versus central levels of the investigated biomarker can be examined. In addition, highly homogeneous samples are available and manipulations are easier than in clinical settings. For this purpose, it is important that the animal model is regarded as highly relevant in the first place. A few examples are following.

The selection of genes for a human pharmacogenetic study in humans was founded on results obtained in a preliminary study in a MDD animal model in response to antidepressants [68]. Mice exposed to maternal separation and chronic mild stress were treated with antidepressants, 
and a transcriptome-wide analysis was performed in hippocampus. Gene expression changes linked with antidepressant treatment were carried forward for testing in a human pharmacogenetic analysis, allowing the identification of the role of a previously unexplored gene (protein phosphatase 1A). Analogous approaches were adopted by Andrus et al. [69] and Pajer et al. [70]. The selection of genes to be tested in humans was established on findings acquired in investigations performed in mouse models. The models tried to reproduce both the genetic and the environmental components of MDD. These components were represented by a substrain identified by selective breeding form Wistar Kyoto rats, which showed increased immobility in the forced swim test, and by the application of chronic restraint stress. The overlap between differentially expressed transcripts in hippocampus, amygdala, and blood was established to arrive at a set of candidate transcripts whose abundance was measured in the blood of human subjects. A panel of blood biomarkers allowed the differentiation of early-onset MDD, whereas a partly overlapping set of transcripts differentiated MDD endophenotypes with or without comorbid anxiety disorders.

Jacobsen et al. [71] aimed at investigating whether the anomalies in putative serotonin biomarkers reported in depressive patients reflected deficient central serotonin neurotransmission. Therefore, they utilised tryptophan hydroxylase 2 loss-of-function mice, which show markedly reduced serotonin synthesis and tissue levels and exhibit increased depression-, anxiety-, and aggression-like behaviours. In this model, it could be demonstrated that peripheral biomarker dysregulations as observed in depressive patients, such as low corticospinal fluid levels of 5-hydroxyindoleacetic acid and blunted fenfluramine-induced prolactin response, were generated by chronic endogenous serotonin deficiency.

Analyses aimed at the discovery of new biomarkers carried out in relevant animal models reaching different stages of validation in humans were discussed in a recent review [72].

Potential indications for future biomarkers studies in patients were also proposed by investigations in a geneenvironment interaction model including antidepressant treatment [73]. Affected analytes included inflammatory mediators and lipid metabolism proteins.

Peripheral biomarkers do not necessarily focus on blood. In a report by Zheng et al. [74], metabolic profiling of urine was performed in a rat model of chronic unpredictable mild stress. Principal component analysis displayed a separation between groups based mainly on levels of analytes related to the disturbance in energy metabolism, amino acid metabolism and gut microflora.

Related to this application are preclinical studies aimed at discovering whether a correlation exists between peripheral and brain expression levels of proteins considered as potentially relevant biomarkers in humans. As an example, several recent investigations analysed neurotrophic factor levels in brain and blood of rats in genetic and environmental models of depression or after treatment [75-77]. Results showed that the correlation can be complex, suggesting that peripheral changes observed in MDD patients should be interpreted cautiously [75-77].

\section{Preclinical Biomarkers to Identify New Pharmacological Targets}

Another objective for measuring peripheral analyte levels in animals is to add this measure to behavioural tests as an additional predictor of efficacy in humans. To be considered as relevant, the investigated biomarker must show a correlation with response to therapy in patients.

When the MDD model relies on the application of stress to provoke depressive-like behaviours, the measure of peripheral stress hormones is often added as a significant end point to evaluate antidepressant activity. Indeed, the dysregulation of stress hormone levels is a frequent feature of depressive patients [78, 79]. A few examples of this kind of studies are following.

In order to evaluate the antidepressant efficacy of melatonin treatment, Detanico et al. [80] utilized a chronic mild stress model in the mouse. Since the model is based on stress exposure, peripheral corticosterone levels were measured as an additional end point with respect to other physiological and behavioural parameters. Maniam and Morris [81] explored the effects of high-fat diet and/or voluntary exercise in behavioural deficits and increased corticosterone response to stress that are induced by exposure to postnatal maternal separation as a model of childhood trauma. Beneficial effects of treatments in reverting maternal separationinduced effects could be demonstrated in behavioural as well as biochemical measures. Similarly, Borsonelo et al. [82] analysed the efficacy of dietary omega-3 fatty acids supplementation in a model of prenatal stress exposure in rats. Both depressive-like behaviours and stress-induced corticosterone increases were reduced by treatment. The olfactory bulbectomized rat model was employed in an investigation oriented at elucidating the mechanism of antidepressant activity of the n-3 fatty acid eicosapentaenoic acid [83]. In this model, depressive-like behaviours and reduced nerve growth factor expression in brain were revealed. In addition, the levels of corticosterone, IL- $1 \beta$, and prostaglandin E2 were measured in blood since the dysregulation of these parameters parallelled those observed in depressive patients. Therefore, the ability to revert these neuroendocrine and immune depression-related modifications was deemed relevant in the analysis of the mechanism of the antidepressant action.

In other models, depressive-like behaviours are provoked by the activation of the immune response. When these models are adopted to investigate antidepressant actions, the reversal of the increase of peripheral inflammation mediators is often added as a relevant end point of the efficacy of treatment. For example, Bison et al. [84] evaluated the effects of a p38 MAP kinase inhibitor in a model based on the stimulation of the innate immunity response generated by lipopolysaccharide treatment. The levels of peripheral inflammation biomarkers were quantified in parallel with behavioural assessments [84]. In addition, to investigate the mechanism of the antidepressant efficacy of exercise, the 
peripheral levels of neuroinflammatory factors were considered as highly significant in understanding the pathogenesis of the disease [85].

\section{Preclinical Biomarkers to Investigate Molecular Mechanisms of Action of Antidepressants}

Another use for peripheral analyte measures in preclinical studies is related to gaining a deeper understanding of the neurobiological basis of efficacious antidepressant activity. It is recognized that modifications of monoamine levels in brain are observed a few hours after antidepressant treatments. In contrast, beneficial effects in terms of mood and other symptoms require at least one week. Therefore, it is likely that additional neurobiological modifications are necessary to obtain the therapeutic benefits $[6,7]$. Increasing this comprehension could lead to new medicines with a different profile as compared to current antidepressants. Hence, a high number of studies are dedicated to this objective and some of them also include the measuring of peripheral analyte levels.

For instance, Behr et al. [86] reviewed the available evidence linking the importance of antioxidant activity in the action of antidepressant medications in both preclinical models and clinical literature. The evaluation of proteins or analytes involved in oxidant or antioxidant effects in the periphery was reported in addition to effects measured in the central nervous system. In addition, the anti-inflammatory activities of antidepressant medications assessed by measuring inflammation mediators were reviewed by Maes et al. [24]. As another example, Watanabe et al. [87] investigated the effect of antidepressants on serum levels of BDNF to explore the hypothesis that therapeutic effects of antidepressants are determined by actions on neurotrophic factors [88]. Results showing that antidepressant treatment induced BDNF release from platelets lent support to the postulated central role of neurotrophic activity, also suggesting BDNF as a putative biomarker of adequate clinical response.

\section{Conclusions}

Since MDD is posing a heavy burden of disease and mortality, utmost efforts should be carried out to understand the neurobiological basis of the disease. This comprehension could help to prevent its occurrence and support the discovery of new therapeutic options. The identification of appropriate biomarkers for human patients is needed to aid diagnosis and therapy. Biomarker studies in animal models of disease can provide a contribution to both the identification of putative clinical biomarkers and to the elucidation of MDD pathophysiology. Indeed, most preclinical studies assessing the peripheral levels of analytes as biomarkers are investigating if the animal model used is relevant to MDD pathophysiology. Peripheral analytes are measured to validate the animal model by showing that stress hormones or immune analytes are dysregulated in the experimental setting in the same direction as in depressive patients. Adding these measures allows a more complete characterisation of the model, with the ultimate objective of improving the understanding of the neurobiological basis of MDD. In contrast, other preclinical studies of peripheral analytes aim at translating the results in clinical settings as a subsequent step. For example, largescale, hypothesis-free studies are carried out in MDD models to pinpoint candidate genes or proteins to be further validated in clinical studies. Other studies investigate putative new targets for drug discovery. Animal models of disease are used for their predictive validity, and the assessment of peripheral analytes is included as an additional measure to increase the confidence in the target. Similarly to the validation studies, stress hormones or immune molecules are usually examined because of their established link with MDD. Moreover, since the mechanism of action of available antidepressants is incompletely understood, several studies investigate the neurobiological alterations associated with antidepressant treatment at a molecular level. While most analyses are centred on brain, in some instances peripheral analytes are also assessed.

Within these different experimental designs, preclinical investigations showed the potential to provide important contributions also in the field of peripheral biomarkers, both to identify clinical biomarkers and to increase our comprehension of MDD.

\section{Abbreviations}

BDNF: Brain-derived neurotrophic factor

HPA: Hypothalamic-pituitary-adrenal axis

MDD: Major depressive disorder.

\section{References}

[1] R. C. Kessler, P. Berglund, O. Demler et al., "The epidemiology of major depressive disorder: results from the national comorbidity survey replication (NCS-R)," The Journal of the American Medical Association, vol. 289, no. 23, pp. 3095-3105, 2003.

[2] H. U. Wittchen, F. Jacobi, J. Rehm et al., "The size and burden of mental disorders and other disorders of the brain in Europe 2010," European Neuropsychopharmacology, vol. 21, no. 9, pp. 655-679, 2011.

[3] A. Halaris, "Comorbidity between depression and cardiovascular disease," International Angiology, vol. 28, no. 2, pp. 92-99, 2009.

[4] B. N. Renn, L. Feliciano, and D. L. Segal, "The bidirectional relationship of depression and diabetes: a systematic review," Clinical Psychology Review, vol. 31, no. 8, pp. 1239-1246, 2011.

[5] American Psychiatric Association, Diagnostic and Statistical Manual of Mental Disorders, Text Revision, American Psychiatric Association, Washington DC, USA, 4th edition, 2000.

[6] A. Baudry, S. Mouillet-Richard, J. Launay, and O. Kellermann, "New views on antidepressant action," Current Opinion in Neurobiology, vol. 21, no. 6, pp. 858-865, 2011.

[7] R. S. Duman and B. Voleti, "Signaling pathways underlying the pathophysiology and treatment of depression: novel mechanisms for rapid-acting agents," Trends in Neurosciences, vol. 35, no. 1, pp. 47-56, 2012.

[8] A. T. Beck and D. J. A. Dozois, "Cognitive therapy: current status and future directions," Annual Review of Medicine, vol. 62, pp. 397-409, 2011. 
[9] M. F. de Mello, J. de Jesus Mari, J. Bacaltchuk, H. Verdeli, and R. Neugebauer, "A systematic review of research findings on the efficacy of interpersonal therapy for depressive disorders," European Archives of Psychiatry and Clinical Neuroscience, vol. 255, no. 2, pp. 75-82, 2005.

[10] The UK ECT Review Group, "Efficacy and safety of electroconvulsive therapy in depressive disorders: a systematic review and meta-analysis," The Lancet, vol. 361, no. 9360, pp. 799-808, 2003.

[11] F. Padberg and M. S. George, "Repetitive transcranial magnetic stimulation of the prefrontal cortex in depression," Experimental Neurology, vol. 219, no. 1, pp. 2-13, 2009.

[12] G. E. Mead, W. Morley, P. Campbell, C. A. Greig, M. McMurdo, and D. A. Lawlor, "Exercise for depression," Cochrane Database of Systematic Reviews, no. 4, Article ID CD004366, 2008.

[13] H. Giedke and F. Schwäzler, "Therapeutic use of sleep deprivation in depression," Sleep Medicine Reviews, vol. 6, no. 5, pp. 361377, 2002.

[14] J. C. Fournier, R. J. DeRubeis, S. D. Hollon et al., "Antidepressant drug effects and depression severity: a patient-level metaanalysis," The Journal of the American Medical Association, vol. 303, no. 1, pp. 47-53, 2010.

[15] A. Fekadu, S. C. Wooderson, K. Markopoulo, C. Donaldson, A. Papadopoulos, and A. J. Cleare, "What happens to patients with treatment-resistant depression? A systematic review of medium to long term outcome studies," Journal of Affective Disorders, vol. 116, no. 1-2, pp. 4-11, 2009.

[16] M. L. Wong and J. Licinio, "From monoamines to genomic targets: a paradigm shift for drug discovery in depression," Nature Reviews Drug Discovery, vol. 3, no. 2, pp. 136-151, 2004.

[17] G. Griebel and F. Holsboer, "Neuropeptide receptor ligands as drugs for psychiatric diseases: the end of the beginning?" Nature Reviews Drug Discovery, vol. 11, no. 6, pp. 462-478, 2012.

[18] R. H. Belmaker and G. Agam, "Major depressive disorder," The New England Journal of Medicine, vol. 358, no. 1, pp. 55-68, 2008.

[19] H. K. Manji, W. C. Drevets, and D. S. Charney, "The cellular neurobiology of depression," Nature Medicine, vol. 7, no. 5, pp. 541-547, 2001.

[20] V. Krishnan and E. J. Nestler, “The molecular neurobiology of depression," Nature, vol. 455, no. 7215, pp. 894-902, 2008.

[21] G. Hasler, W. C. Drevets, H. K. Manji, and D. S. Charney, "Discovering endophenotypes for major depression," Neuropsychopharmacology, vol. 29, no. 10, pp. 1765-1781, 2004.

[22] E. Palazidou, "The neurobiology of depression," The British Medical Bulletin, vol. 101, no. 1, pp. 127-145, 2012.

[23] E. Castrén, V. Võikar, and T. Rantamäki, "Role of neurotrophic factors in depression," Current Opinion in Pharmacology, vol. 7, no. 1, pp. 18-21, 2007.

[24] M. Maes, R. Yirmyia, J. Noraberg et al., "The inflammatory \& neurodegenerative (I\&ND) hypothesis of depression: leads for future research and new drug developments in depression," Metabolic Brain Disease, vol. 24, no. 1, pp. 27-53, 2009.

[25] N. Kronfeld-Schor and H. Einat, "Circadian rhythms and depression: human psychopathology and animal models," Neuropharmacology, vol. 62, no. 1, pp. 101-114, 2012.

[26] L. Capuron and R. Dantzer, "Cytokines and depression: the need for a new paradigm," Brain, Behavior, and Immunity, vol. 17, supplement 1, pp. S119-S124, 2003.

[27] R. Yirmiya and I. Goshen, "Immune modulation of learning, memory, neural plasticity and neurogenesis," Brain, Behavior, and Immunity, vol. 25, no. 2, pp. 181-213, 2011.
[28] C. Song and H. Wang, "Cytokines mediated inflammation and decreased neurogenesis in animal models of depression," Progress in Neuro-Psychopharmacology and Biological Psychiatry, vol. 35, no. 3, pp. 760-768, 2011.

[29] H. Anisman and Z. Merali, "Cytokines, stress and depressive illness: brain-immune interactions," Annals of Medicine, vol. 35, no. 1, pp. 2-11, 2003.

[30] A. H. Miller, V. Maletic, and C. L. Raison, "Inflammation and its discontents: the role of cytokines in the pathophysiology of major depression," Biological Psychiatry, vol. 65, no. 9, pp. 732741, 2009.

[31] H. D. Schmidt, R. C. Shelton, and R. S. Duman, "Functional biomarkers of depression: diagnosis, treatment, and pathophysiology," Neuropsychopharmacology, vol. 36, no. 12, pp. 23752394, 2011.

[32] A. F. Leuchter, I. A. Cook, S. P. Hamilton et al., "Biomarkers to predict antidepressant response," Current Psychiatry Reports, vol. 12, no. 6, pp. 553-562, 2010.

[33] M. Lekman, S. Paddock, and F. J. McMahon, "Pharmacogenetics of major depression: Insights from level 1 of the sequenced treatment alternatives to relieve depression $\left(\mathrm{STAR}^{*} \mathrm{D}\right)$ trial," Molecular Diagnosis and Therapy, vol. 12, no. 5, pp. 321-330, 2008.

[34] M. J. Taylor, N. Freemantle, J. R. Geddes, and Z. Bhagwagar, "Early onset of selective serotonin reuptake inhibitor antidepressant action: systematic review and meta-analysis," Archives of General Psychiatry, vol. 63, no. 11, pp. 1217-1223, 2006.

[35] G. I. Papakostas, R. H. Perlis, M. J. Scalia, T. J. Petersen, and M. Fava, "A meta-analysis of early sustained response rates between antidepressants and placebo for the treatment of major depressive disorder," Journal of Clinical Psychopharmacology, vol. 26, no. 1, pp. 56-60, 2006.

[36] A. Steiger and M. Kimura, "Wake and sleep EEG provide biomarkers in depression," Journal of Psychiatric Research, vol. 44, no. 4, pp. 242-252, 2010.

[37] M. P. Quinones and R. Kaddurah-Daouk, "Metabolomics tools for identifying biomarkers for neuropsychiatric diseases," Neurobiology of Disease, vol. 35, no. 2, pp. 165-176, 2009.

[38] O. Berton, C. G. Hahn, and M. E. Thase, "Are we getting closer to valid translational models for major depression?" Science, vol. 338, no. 6103, pp. 75-79, 2012.

[39] B. A. Samuels, E. D. Leonardo, R. Gadient et al., "Modeling treatment-resistant depression," Neuropharmacology, vol. 61, no. 3, pp. 408-413, 2011.

[40] J. F. Cryan and D. A. Slattery, "Animal models of mood disorders: recent developments," Current Opinion in Psychiatry, vol. 20, no. 1, pp. 1-7, 2007.

[41] E. J. Nestler and S. E. Hyman, "Animal models of neuropsychiatric disorders," Nature Neuroscience, vol. 13, no. 10, pp. 1161$1169,2010$.

[42] M. F. O’Neil and N. A. Moore, "Animal models of depression: are there any?" Human Psychopharmacology, vol. 18, no. 4, pp. 239-254, 2003.

[43] C. R. Pryce and E. Seifritz, "A translational research framework for enhanced validity of mouse models of psychopathological states in depression," Psychoneuroendocrinology, vol. 36, no. 3, pp. 308-329, 2011.

[44] M. V. Schmidt, X. Wang, and O. C. Meijer, "Early life stress paradigms in rodents: potential animal models of depression?" Psychopharmacology, vol. 214, no. 1, pp. 131-140, 2011. 
[45] C. Song and B. E. Leonard, "The olfactory bulbectomised rat as a model of depression," Neuroscience and Biobehavioral Reviews, vol. 29 , no. $4-5$, pp. $627-647,2005$.

[46] D. H. Overstreet, E. Friedman, A. A. Mathé, and G. Yadid, "The flinders sensitive line rat: a selectively bred putative animal model of depression," Neuroscience and Biobehavioral Reviews, vol. 29, no. 4-5, pp. 739-759, 2005.

[47] F. A. Henn and B. Vollmayr, "Stress models of depression: forming genetically vulnerable strains," Neuroscience and Biobehavioral Reviews, vol. 29, no. 4-5, pp. 799-804, 2005.

[48] R. Dantzer, J. C. O’Connor, M. A. Lawson, and K. W. Kelley, "Inflammation-associated depression: from serotonin to kynurenine," Psychoneuroendocrinology, vol. 36, no. 3, pp. 426436, 2011.

[49] M. Maes, M. Berk, L. Goehler et al., "Depression and sickness behavior are Janus-faced responses to shared inflammatory pathways," BMC Medicine, vol. 10, article 66, 2012.

[50] H. Abe, N. Hidaka, C. Kawagoe et al., "Prenatal psychological stress causes higher emotionality, depression-like behavior, and elevated activity in the hypothalamo-pituitary-adrenal axis," Neuroscience Research, vol. 59, no. 2, pp. 145-151, 2007.

[51] M. Razzoli, E. Roncari, A. Guidi et al., "Conditioning properties of social subordination in rats: behavioral and biochemical correlates of anxiety," Hormones and Behavior, vol. 50, no. 2, pp. 245-251, 2006.

[52] M. Razzoli, L. Carboni, A. Guidi, P. Gerrard, and R. Arban, "Social defeat-induced contextual conditioning differentially imprints behavioral and adrenal reactivity: a time-course study in the rat," Physiology and Behavior, vol. 92, no. 4, pp. 734-740, 2007.

[53] M. Razzoli, L. Carboni, and R. Arban, "Alterations of behavioral and endocrinological reactivity induced by 3 brief social defeats in rats: relevance to human psychopathology," Psychoneuroendocrinology, vol. 34, no. 9, pp. 1405-1416, 2009.

[54] M. Razzoli, L. Carboni, M. Andreoli, A. Ballottari, and R. Arban, "Different susceptibility to social defeat stress of BalbC and C57BL6/J mice," Behavioural Brain Research, vol. 216, no. 1, pp. 100-108, 2011.

[55] M. Razzoli, E. Domenici, L. Carboni et al., "A role for $\mathrm{BDNF} / \mathrm{TrkB}$ signaling in behavioral and physiological consequences of social defeat stress," Genes, Brain and Behavior, vol. 10, no. 4, pp. 424-433, 2011.

[56] N. Bowens, W. Heydendael, S. Bhatnagar, and L. Jacobson, "Lack of elevations in glucocorticoids correlates with dysphoria-like behavior after repeated social defeat," Physiology and Behavior, vol. 105, no. 4, pp. 958-965, 2012.

[57] C. Touma, T. Fenzl, J. Ruschel et al., "Rhythmicity in mice selected for extremes in stress reactivity: behavioural, endocrine and sleep changes resembling endophenotypes of major depression," PLoS ONE, vol. 4, no. 1, Article ID e4325, 2009.

[58] A. Blugeot, C. Rivat, E. Bouvier et al., "Vulnerability to depression: from brain neuroplasticity to identification of biomarkers," Journal of Neuroscience, vol. 31, no. 36, pp. 12889-12899, 2011.

[59] X. Y. Lu, C. S. Kim, A. Frazer, and W. Zhang, "Leptin: a potential novel antidepressant," Proceedings of the National Academy of Sciences of the United States of America, vol. 103, no. 5, pp. 15931598, 2006.

[60] J. C. Chuang, V. Krishnan, H. G. Yu et al., "A $\beta 3$-adrenergicleptin-melanocortin circuit regulates behavioral and metabolic changes induced by chronic stress," Biological Psychiatry, vol. 67, no. 11, pp. 1075-1082, 2010.
[61] A. C. D’Audiffret, S. J. Frisbee, P. A. Stapleton, A. G. Goodwill, E. Isingrini, and J. C. Frisbee, "Depressive behavior and vascular dysfunction: a link between clinical depression and vascular disease?" Journal of Applied Physiology, vol. 108, no. 5, pp. 10411051, 2010.

[62] J. C. O'Connor, M. A. Lawson, C. André et al., "Lipopolysaccharide-induced depressive-like behavior is mediated by indoleamine 2,3-dioxygenase activation in mice," Molecular Psychiatry, vol. 14, no. 5, pp. 511-522, 2009.

[63] J. C. O'Connor, C. André, Y. Wang et al., "Interferon- $\gamma$ and tumor necrosis factor- $\alpha$ mediate the upregulation of indoleamine 2,3-dioxygenase and the induction of depressivelike behavior in mice in response to bacillus calmette-guérin," Journal of Neuroscience, vol. 29, no. 13, pp. 4200-4209, 2009.

[64] G. Naert, G. Ixart, T. Maurice, L. Tapia-Arancibia, and L. Givalois, "Brain-derived neurotrophic factor and hypothalamicpituitary-adrenal axis adaptation processes in a depressive-like state induced by chronic restraint stress," Molecular and Cellular Neuroscience, vol. 46, no. 1, pp. 55-66, 2011.

[65] F. Cirulli, N. Francia, I. Branchi et al., "Changes in plasma levels of BDNF and NGF reveal a gender-selective vulnerability to early adversity in rhesus macaques," Psychoneuroendocrinology, vol. 34, no. 2, pp. 172-180, 2009.

[66] A. Moranis, J. Delpech, V. de Smedt-Peyrusse et al., "Long term adequate $n-3$ polyunsaturated fatty acid diet protects from depressive-like behavior but not from working memory disruption and brain cytokine expression in aged mice," Brain, Behavior, and Immunity, vol. 26, no. 5, pp. 721-731, 2012.

[67] L. Capuron, S. Schroecksnadel, C. Féart et al., "Chronic lowgrade inflammation in elderly persons is associated with altered tryptophan and tyrosine metabolism: role in neuropsychiatric symptoms," Biological Psychiatry, vol. 70, no. 2, pp. 175-182, 2011.

[68] K. Malki, R. Uher, J. Paya-Cano et al., "Convergent animal and human evidence suggests a role of PPM1A gene in response to antidepressants," Biological Psychiatry, vol. 69, no. 4, pp. $360-$ 365, 2011.

[69] B. M. Andrus, K. Blizinsky, P. T. Vedell et al., "Gene expression patterns in the hippocampus and amygdala of endogenous depression and chronic stress models," Molecular Psychiatry, vol. 17, no. 1, pp. 49-61, 2012.

[70] K. Pajer, B. M. Andrus, W. Gardner et al., "Discovery of blood transcriptomic markers for depression in animal models and pilot validation in subjects with early-onset major depression," Translational Psychiatry, vol. 2, article e101, 2012.

[71] J. P. R. Jacobsen, W. B. Siesser, B. D. Sachs et al., "Deficient serotonin neurotransmission and depression-like serotonin biomarker alterations in tryptophan hydroxylase 2 (Tph2) lossof-function mice," Molecular Psychiatry, vol. 17, no. 7, pp. 694704, 2012.

[72] Z. Sarnyai, M. Alsaif, S. Bahn et al., "Behavioral and molecular biomarkers in translational animal models for neuropsychiatric disorders," International Review of Neurobiology, vol. 101, pp. 203-238, 2011.

[73] L. Carboni, S. Becchi, C. Piubelli et al., "Early-life stress and antidepressants modulate peripheral biomarkers in a geneenvironment rat model of depression," Progress in NeuroPsychopharmacology and Biological Psychiatry, vol. 34, no. 6, pp. 1037-1048, 2010.

[74] S. Zheng, M. Yu, X. Lu et al., "Urinary metabonomic study on biochemical changes in chronic unpredictable mild stress model of depression," Clinica Chimica Acta, vol. 411, no. 3-4, pp. 204-209, 2010. 
[75] B. Elfving, P. H. Plougmann, H. K. Müller, A. A. Mathé, R. Rosenberg, and G. Wegener, "Inverse correlation of brain and blood BDNF levels in a genetic rat model of depression," International Journal of Neuropsychopharmacology, vol. 13, no. 5, pp. 563-572, 2010.

[76] A. Sartorius, R. Hellweg, J. Litzke et al., "Correlations and discrepancies between serum and brain tissue levels of neurotrophins after electroconvulsive treatment in rats," Pharmacopsychiatry, vol. 42, no. 6, pp. 270-276, 2009.

[77] K. R. Luo, C. J. Hong, Y. J. Liou, S. J. Hou, Y. H. Huang, and S. J. Tsai, "Differential regulation of neurotrophin S100B and BDNF in two rat models of depression," Progress in NeuroPsychopharmacology and Biological Psychiatry, vol. 34, no. 8, pp. 1433-1439, 2010.

[78] C. M. Pariante and S. L. Lightman, "The HPA axis in major depression: classical theories and new developments," Trends in Neurosciences, vol. 31, no. 9, pp. 464-468, 2008.

[79] C. F. Gillespie and C. B. Nemeroff, "Hypercortisolemia and depression," Psychosomatic Medicine, vol. 67, supplement 1, pp. S26-S28, 2005.

[80] B. C. Detanico, A. L. Piato, J. J. Freitas et al., "Antidepressant-like effects of melatonin in the mouse chronic mild stress model," European Journal of Pharmacology, vol. 607, no. 1-3, pp. 121-125, 2009.

[81] J. Maniam and M. J. Morris, "Voluntary exercise and palatable high-fat diet both improve behavioural profile and stress responses in male rats exposed to early life stress: role of hippocampus," Psychoneuroendocrinology, vol. 35, no. 10, pp. 1553-1564, 2010.

[82] E. C. Borsonelo, D. Suchecki, and J. C. F. Galduróz, "Effect of fish oil and coconut fat supplementation on depressive-type behavior and corticosterone levels of prenatally stressed male rats," Brain Research, vol. 1385, pp. 144-150, 2011.

[83] C. Song, Y. Z. Xiang, and M. Manku, "Increased phospholipase A2 activity and inflammatory response but decreased nerve growth factor expression in the olfactory bulbectomized rat model of depression: effects of chronic ethyl-eicosapentaenoate treatment," Journal of Neuroscience, vol. 29, no. 1, pp. 14-22, 2009.

[84] S. Bison, M. Razzoli, R. Arban, F. Michielin, S. Bertani, and L. Carboni, "Effect of the p38 MAPK inhibitor SB-239063 on Lipopolysaccharide-induced psychomotor retardation and peripheral biomarker alterations in rats," European Journal of Pharmacology, vol. 661, no. 1-3, pp. 49-56, 2011.

[85] H. Eyre and B. T. Baune, "Neuroimmunological effects of physical exercise in depression," Brain, Behavior, and Immunity, vol. 26, no. 2, pp. 251-266, 2012.

[86] G. A. Behr, J. C. Moreira, and B. N. Frey, "Preclinical and clinical evidence of antioxidant effects of antidepressant agents: implications for the pathophysiology of major depressive disorder," Oxidative Medicine and Cellular Longevity, vol. 2012, Article ID 609421, 13 pages, 2012.

[87] K. Watanabe, E. Hashimoto, W. Ukai et al., "Effect of antidepressants on brain-derived neurotrophic factor (BDNF) release from platelets in the rats," Progress in NeuroPsychopharmacology and Biological Psychiatry, vol. 34, no. 8, pp. 1450-1454, 2010.

[88] R. S. Duman and L. M. Monteggia, "A neurotrophic model for stress-related mood disorders," Biological Psychiatry, vol. 59, no. 12, pp. 1116-1127, 2006. 


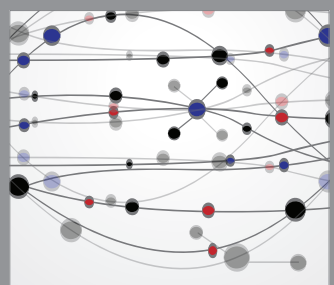

The Scientific World Journal
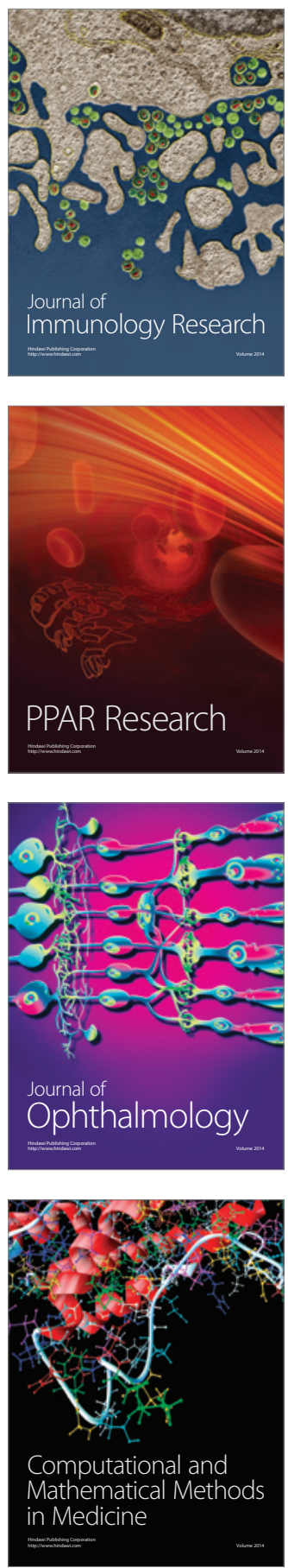

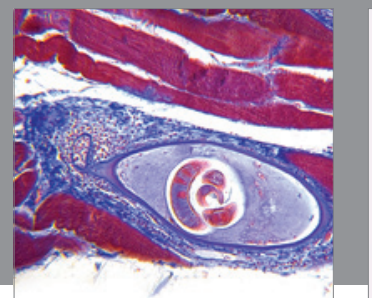

Gastroenterology

Research and Practice
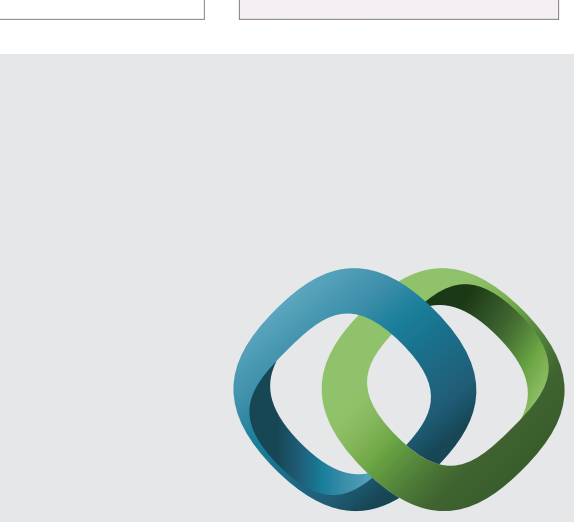

\section{Hindawi}

Submit your manuscripts at

http://www.hindawi.com
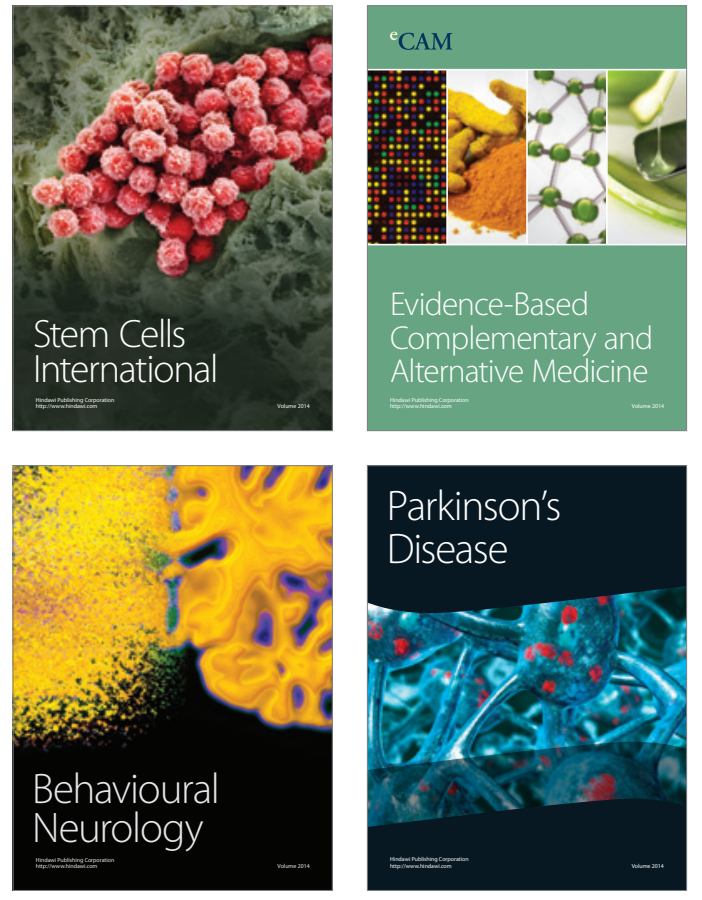
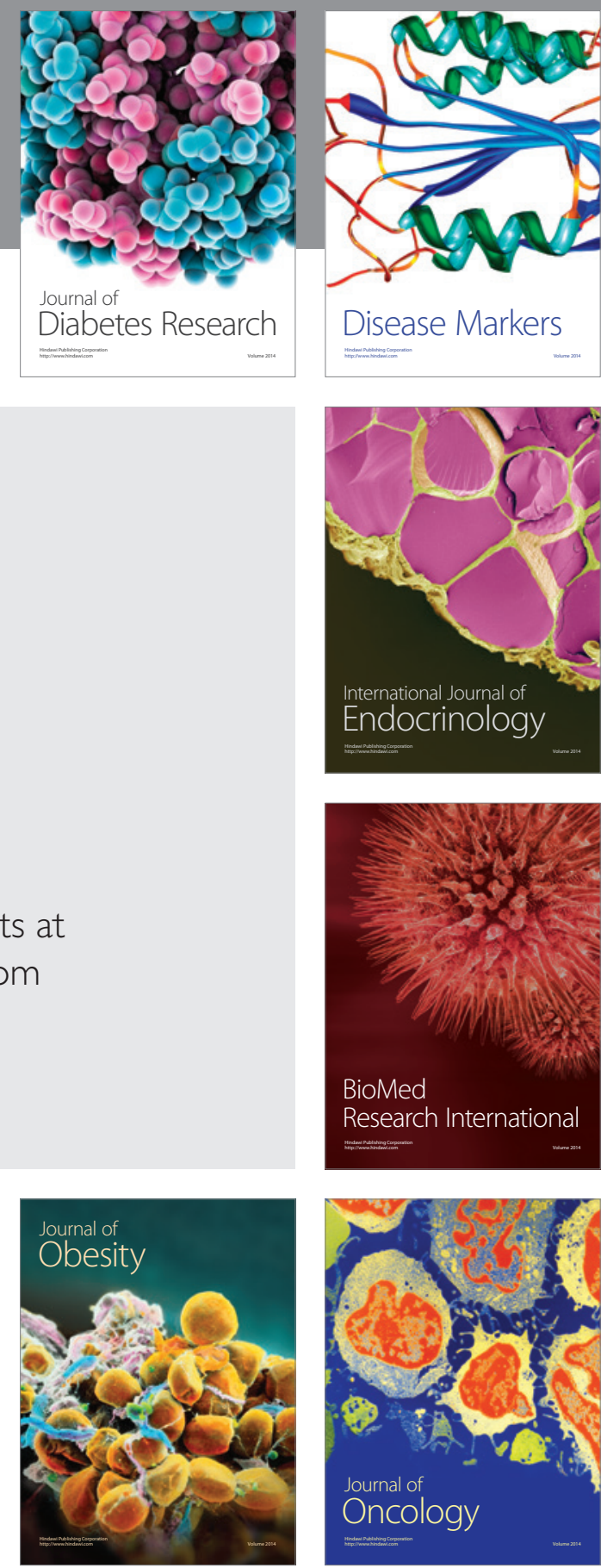

Disease Markers
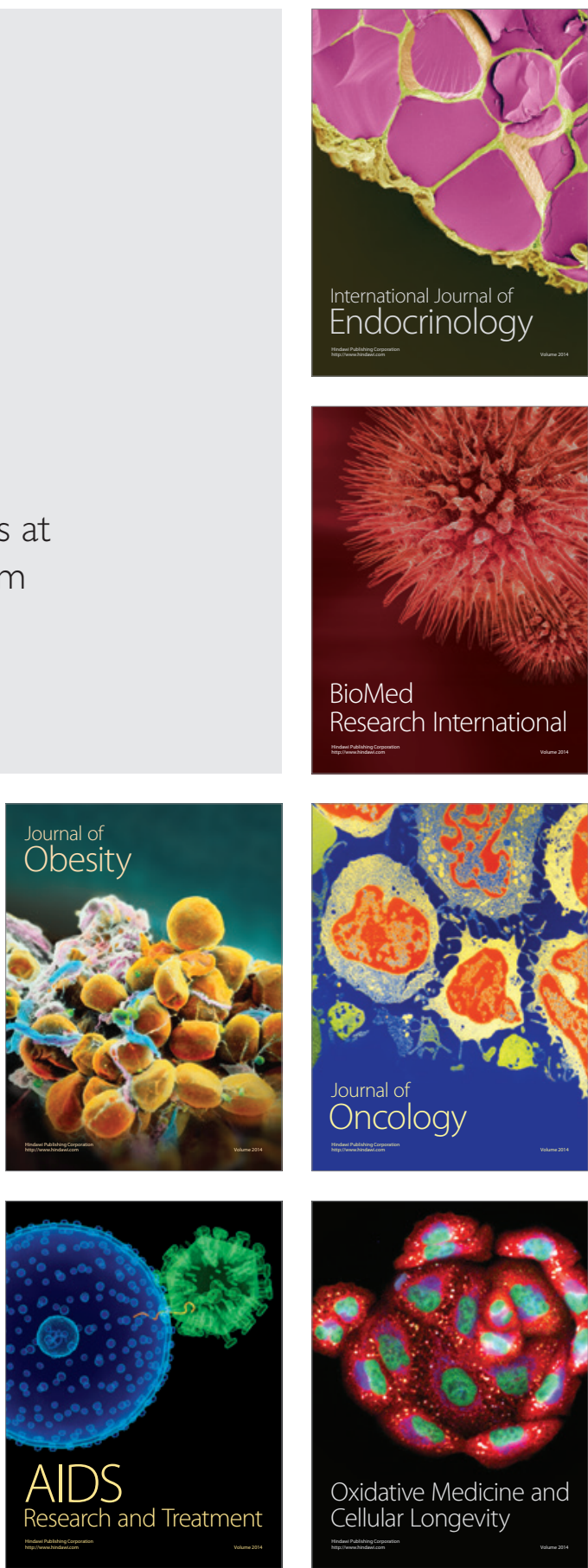